\title{
Observing repetitive finger movements modulates response times of auditorily cued finger movements
}

Biermann-Ruben, K., Jonas, M., Kessler, K., Siebner, H. R., Bäumer, T., Schnitzler, A. \& Münchau

\section{Introduction}

What we see has a great impact on what we do or how fast we react, even if what we see is irrelevant to what we are required to do. The degree of correspondence between the characteristics of the stimulus and the response afforded by the task has a substantial influence on the selection and generation of the response and is commonly referred to as stimulus-response compatibility (SRC) (Fitts \& Seeger, 1953). In the particular case that both stimuli and required responses constitute motor actions, their correspondence has been specified by the related concept of ideomotor compatibility (Greenwald, 1972 and James, 1890).

Using a stimulus-response compatibility paradigm, Brass, Bekkering, Wohlschläger, and Prinz (2000) showed that the observation of irrelevant biological movements (i.e., finger movements) strongly influenced reaction times (RTs) to both task-relevant symbolic and spatial cues depending on stimulus-response compatibility. In this context the "relevant" stimulus prompts the subject's response, whereas the "irrelevant" stimulus-either being compatible or incompatible to the relevant stimulus (and the response) has no informative value concerning the task. Taskirrelevant symbolic cues had no effect on imitative responses to biological cues, whereas irrelevant incompatible spatial cues slowed down reactions to biological cues. They also found that RTs to biological movement cues were on average $91 \mathrm{~ms}$ shorter than reactions to symbolic cues and $41 \mathrm{~ms}$ shorter than reactions to spatial cues. Although RT changes related to the observation of finger movements are in part caused by spatial stimulus-response compatibility, automatic imitation processes still make a significant contribution (Bertenthal, Longo, \& Kosobud, 2006). Interestingly, it was shown that the effects of automatic imitation declined with time while effects of spatial compatibility remained constant or increased slightly during the experimental session (Bertenthal et al., 2006). The authors concluded that different processes underlie both effects. Extending these findings, Jonas et al. (2007a) showed that in a spatial choice RT task, responses were still faster to biological finger movements than to spatially and kinematically matched dot movements.

Neuroscientists recently identified the mirror neuron system (MNS) as a possible cortical substrate that mediates the automatic imitation of actions. First evidence for the existence of an MNS resulted from single unit recordings of neurons in the macaque monkeys' ventral premotor area F5 and inferior parietal area PF (di Pellegrino et al., 1992, Fogassi et al., 2005, Gallese et al., 1996 and Rizzolatti et al., 1996). Those neurons are similarly active irrespective of whether the monkey performs an action or if the monkey observes another individual performing this action. Numerous neuroimaging studies supported the assumption that a similar system also exists in humans (for a review see (lacoboni \& Dapretto, 2006)). This "mirror activity" has also been referred to as action observation-execution matching (AOEM). It has been proposed that the activation of motor related areas during action observation subserves the process of understanding the action (Hari and Nishitani, 2004 and Rizzolatti, 2004) by accessing the motor repertoire of the observer (Gallese, Keysers, \& Rizzolatti, 2004).

Since several studies showed that biological stimuli are accompanied by faster reaction times as compared with nonbiological stimuli (Bertenthal et al., 2006, Brass et al., 2000 and Jonas et al., 2007a) and that stimulus-response compatibility also facilitates responses in settings requiring immediate responses, the main focus of the present study was to address the respective influence of these two factors and their interaction. We were particularly interested to examine whether response facilitation critically depends on the temporal relationship between movement observation and response initiation and whether this relationship is also modulated by the duration of movement observation. To this end, healthy human subjects performed an auditory two-choice reaction time (RT) task, which required a rapid extension-flexion movement of the index or little finger. Participants observed repetitive movements of a finger or dot during the RT task. In two separate experiments, participants observed a long (8 to 11 
repeats) or short (1 to 2 repeats) movement sequence. Movements were either congruent or incongruent to the motor response required by a tone. This enabled us to test whether the matching of action observation and execution was modified by the duration of movement observation (short versus long movement sequence) or the coincidence of action observation and execution (onset versus offset stimulation).

\section{Experiment 1}

\subsection{Materials and methods}

\subsubsection{Subjects}

Sixteen healthy male subjects aged between 23 and 36 years (mean 28 years) participated in this experiment. All were consistently right handed as assessed with the revised Annett Handedness Questionnaire (Annett, 1985) and had no history of neuropsychiatric disorder. All subjects had normal vision, were naive with respect to the purpose of the study and signed an informed consent prior to the experiment. The study was in accordance with the Declaration of Helsinki (1964) and approved by the Ethics Committee of the University Medical Center HamburgEppendorf (No. OB/6/02 (1686)).

\subsubsection{Visual and auditory stimuli}

Visual stimuli were the same as used in Jonas et al. (2007a). The basic visual stimulus was a colour picture of a left male hand resting on a white horizontal plane with fingers slightly flexed (Fig. 1). All subjects were instructed to respond with their right hand during the experiment. The visual stimulus was presented in a specular orientation with respect to the responding hand because of evidence for stronger MNS activation in "mirror"-mode (i.e., specular) imitation as compared to anatomically correct imitation (Koski, lacoboni, Dubeau, Woods, \& Mazziotta, 2003). In addition, we wanted to avoid effects due to spatial incompatibility of stimuli and responses as described by Bertenthal et al. (Bertenthal et al., 2006). The fingernails of the index and little finger were labelled with red dots of approximately $1 \mathrm{~cm}$ diameter. On the horizontal midline of the visual stimulus and with equal distance to the tip of the index and little finger a white cross was presented, which the subjects were required to fixate during the experiment.
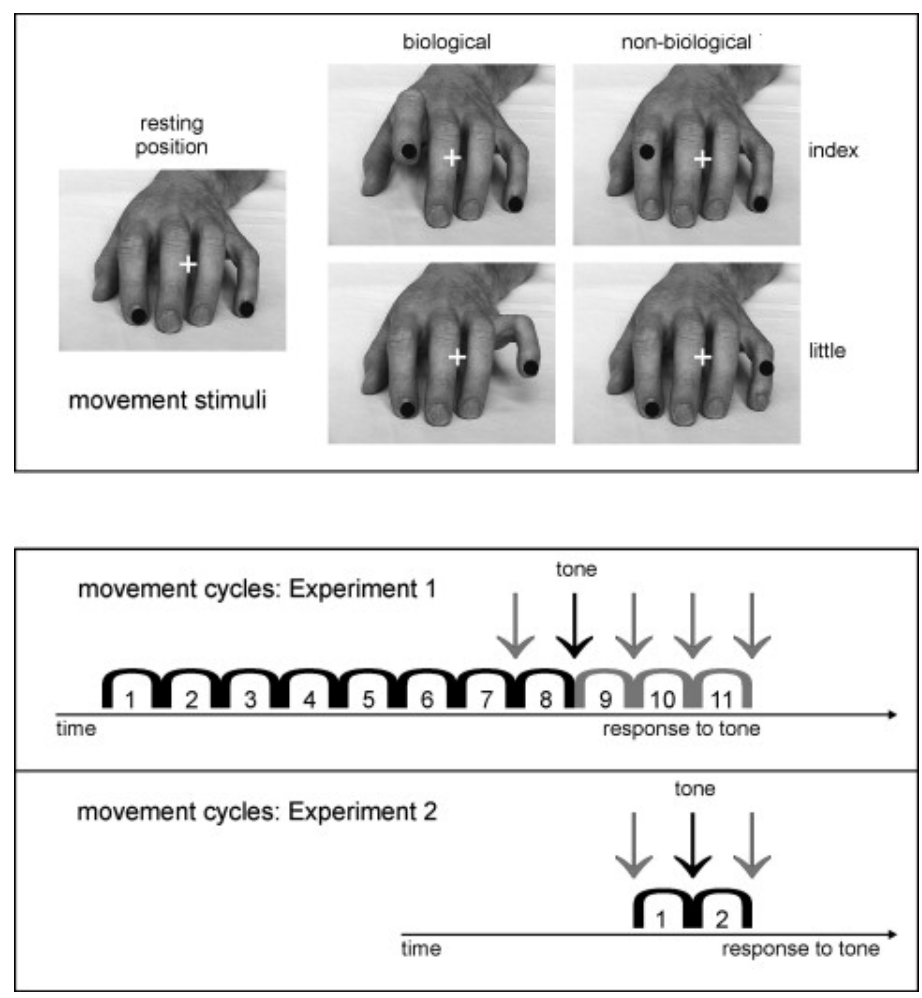

Fig. 1. 
Movement stimuli and movement cycles used in Experiment 1 and 2. Movement stimuli: five picture frames characterize the resting hand position and the stimulus conditions biological vs. non-biological movement of index and little finger, respectively. The four plotted picture frames on the right side depict the maximum amplitude of the movement. Measures of the visual stimuli were $24.5^{\circ}$ diagonally (whole frame), $9.3^{\circ}$ between dots and $6^{\circ}$ from each dot to the fixation cross (all measures center to center during rest). Pictures were presented in colour during the experiment and movements were perceived as continuous up-and-down finger taps. Movement cycles: one arc represents one movement (up-down). In Experiment 1, up to eleven cycles were presented, while there were one or two cycles in Experiment 2. One exemplary condition per experiment is plotted in black: eight cycles with tone presentation at movement offset (Experiment 1 ) and two cycles with tone presentation at movement onset (Experiment 2). All other conditions are plotted in grey.

There were two types of movement stimuli: biological finger movements consisted of eight to eleven repetitive upand-down movements (compare Fig. 1) of the index or little finger including the corresponding dot. In the nonbiological movement sequence, the finger remained stationary and one of the red dots moved up and down with the same kinematical profile as during the biological movement, ensuring that it always moved on the corresponding finger but not next to it. A single up-and-down movement lasted $433 \mathrm{~ms}$ comprising 13 picture frames of about $33 \mathrm{~ms}$ each (two refresh rates of the computer monitor). Subjects were seated at a viewing distance of $1 \mathrm{~m}$ in front of a screen, and visual stimuli were backward projected. Visual angles of the stimulus comprised approximately 24.5

- for the diagonal of the complete picture frame. During the resting position the distance between both dots that labelled the fingers measured $9.3^{\circ}$ (compare Fig. 1), whereas the distance of each dot to the fixation cross was $6^{\circ}$. Auditory stimuli consisted of single sinusoidal tones of either $500 \mathrm{~Hz}$ (low tone) or $1000 \mathrm{~Hz}$ (high tone) of $50 \mathrm{~ms}$ duration including $10 \mathrm{~ms}$ rise and fall. Subjects heard the tones binaurally via plastic tubes.

\subsubsection{Experimental procedure and task}

Participants were seated in an acoustically shielded room with dimmed light. In a first run subjects observed the static hand stimulus and occasionally heard a low or high-frequency tone. The tone coded, which finger the subjects had to lift: 8 subjects lifted the index finger to the low tone and the little finger to the high tone, to the other 8 subjects the opposite instruction was given. As soon as they knew which tone was the high/low one they responded as fast as possible with the corresponding finger. This tone-finger-association run comprised 20 trials per finger, i.e., 40 trials in total. In the second run subjects performed a baseline RT-measurement consisting of 20 trials per finger, again observing the static hand stimulus. The subjects' right hand was placed in an opto-electronic device that responded to upward movements of the index and little finger. Light barriers (one for each finger) were attached to a plane and index and little finger were positioned cutting the lights. The light barrier reacted as soon as a finger left the plane (lift). Reaction times (RTs) were calculated from the onset of a tone to the subjects' lift response. False responses (omissions, false single responses, multiple responses) were marked. In a third run, the practice run, subjects performed 15 trials comprising different conditions of the main measurement as described below.

In the main experiment subjects observed sequences of short repetitive movements, which terminated with the presentation of the resting hand. Additionally, they heard the same low or high-frequency tone to which they responded during the baseline measurement. Repetitive movements lasted between 3464 and 4763 ms according to the amount of repetitions ( 8 to 11). The tone was presented either at the onset of the last movement repetition (onset presentation mode) or after the last movement was completed (offset presentation mode). After the last movement of a sequence, the static hand was presented until completion of the pre-set trial duration of $7000 \mathrm{~ms}$. Again, false responses were marked.

\subsubsection{Experimental design}

The experiment had a 2 (type of movement) $\times 2$ (congruency) $\times 2$ (presentation mode) factorial design. The type of movement was either biological or non-biological. Congruency referred to the correspondence of the visual 
movement and the imperative auditory cue (compatible or incompatible). Auditory cues were presented with the onset or offset of the last tapping cycle.

Finger position (index or little finger) was equally distributed across the conditions as was the amount of repetitions. Each of the eight conditions was applied 36 times; we therefore applied a total of 288 valid trials.

The experimental design also included two types of catch trials. The first type of catch trials served to reduce effects of temporal expectancy regarding presentation of the imperative tone. To this end, tones were omitted and subjects were required not to respond. In the second type of catch trial, we presented a movement series, which was "disturbed" by one movement of the other finger (or dot). Here, a tone was presented as in the valid trials, but subjects were again instructed not to respond in these trials. Each type of catch trial was repeated 16 times per session, which corresponds to $10 \%$ of all trials. Stimulus type as well as movement positions were equally distributed across catch trials. We introduced the catch trials to ensure a minimum amount of vigilance during the repeated presentation of irrelevant movement stimuli. All 320 trials were presented randomly in eight blocks with 40 trials each; one block lasted approximately five minutes.

\subsubsection{Statistical evaluation}

Incorrect responses and correct responses with RTs below $100 \mathrm{~ms}$ or above the mean RT value plus the 2.5 -fold standard deviation of the subject's RT distribution were excluded from analysis.

Since our main focus of interest was the interaction between type of movement and congruency in different temporal relationships between movement observation and motor response, reaction times of the main experiment were analysed separately for onset and offset stimulus presentations: for both presentation modes an ANOVA with factors "type of movement" (biological vs. non-biological) and "congruency" (congruent vs. incongruent) was performed.

We hypothesized an effect of congruency with congruent (observed and required) movements leading to faster responses than incongruent movements with both presentation modes. Furthermore, we predicted that nonbiological conditions (i.e., the observation of dot movements) should be less affected by congruency with respect to RTs than biological conditions (i.e., the observation of biological movements). Therefore, we expected an interaction between "type of movement" and "congruency" revealing fastest RTs in congruent biological conditions and slowest RTs in incongruent biological conditions. We further hypothesized that an imitative response facilitation would be present with compatible and a response inhibition with incompatible biological movements provided movement observation and execution occur at the same time, but not if movement execution occurs after observation.

\subsection{Results}

One subject was excluded because he responded to all type 2 catch trials. Error rates of the remaining 15 subjects in valid trials ranged from 1.4 to $12.9 \%$ (mean $6.6 \%$ ). During onset presentation conditions, $80 \%$ of all errors were multiple responses or false single responses, while these error types equalled $79 \%$ during offset presentations. Mean RT for the baseline measurement was $423 \mathrm{~ms}$ (SD $74 \mathrm{~ms}$ ).

Results of the statistical analyses are summarized in Table 1. When tones were presented at the onset of the tapping movement, responses were faster with observation of biological movements relative to non-biological movements (521 ms vs. $535 \mathrm{~ms} ; \mathrm{p}=.022$ ). No such effect was observed with presentation of the tones at the offset of the observed tapping movement ( $510 \mathrm{~ms}$ vs. $519 \mathrm{~ms} ; \mathrm{p}=.281$ ). In the "onset" condition, responses to congruent movements were faster than to incongruent movements though this difference just failed to reach significance (516 ms vs. $539 \mathrm{~ms} ; \mathrm{p}=.057$ ). This effect was significant in trials in which the tone was presented at movement offset (504 vs. $525 \mathrm{~ms} ; \mathrm{p}=.025$ ). The interaction between "type of movement" and "congruency" approached significance in the "onset" condition ( $p=.051$ ) but not in the "offset" condition ( $p=.443)$. In congruent trials, RTs were faster when subjects observed biological stimuli relative to the observation of non-biological stimuli (post hoc 
Scheffé test: $p=.009)$, whereas RTs did not differ between biological and non-biological conditions in incongruent trials (Scheffé-test: $p=.745$ ).

Table 1.

Results of analyses of variance, Experiment 1

Reaction times in milliseconds; means and standard deviations (SD)

\begin{tabular}{|c|c|c|}
\hline \multirow{3}{*}{ Conditions } & \multirow[t]{2}{*}{ Onset presentation } & \multirow[t]{2}{*}{ Offset presentation } \\
\hline & & \\
\hline & \multicolumn{2}{|c|}{ Biological Non-biological Biological Non-biological } \\
\hline Congruent & $505(88) 527(101)$ & 502 (104) 506 (97) \\
\hline Incongruent & $536(95) 542(95)$ & $518(108) 532(102)$ \\
\hline \multicolumn{3}{|c|}{ Effects: $p$-values (F-values) } \\
\hline \multicolumn{2}{|c|}{ Type of movement 0.022 (6.69) } & $0.281(1.26)$ \\
\hline Congruency & $0.057(4.28)$ & $0.025(6.26)$ \\
\hline Interaction & $0.051(4.54)$ & $0.443(0.62)$ \\
\hline
\end{tabular}

Mean reaction times and standard deviations are plotted for biological/non-biological congruent/incongruent trials, and separately for onset presentations and offset presentations. Main effects and interactions of the Anovas typeof-movement $\times$ congruency separately for processing modes are depicted as $p$ - and F-values.

\subsection{Discussion}

Though visual stimulation had no informative value for the subject with respect to task performance in the main experimental conditions, the observation of spatially congruent stimuli shortened mean RTs. This congruency effect most likely represents a typical Simon-effect based on spatiotemporal overlap of stimulus and response sets (Kornblum, Hasbroucq, \& Osman, 1990). Required movements matched finger positions that were visually presented, resulting in spatial SRC (for an overview concerning Simon-effects see (Simon, 1990)). Our results are in good accordance with studies of Stürmer et al. (Stürmer, 1997 and Stürmer et al., 2000) who also found effects of congruency of irrelevant and non-predictive biological movement stimuli. In that study, observed manual gestures led to RT advantages for concurrent execution of congruent as compared with incongruent manual gestures that were instructed by a colour change.

RTs were faster with congruent biological than with non-biological stimuli. This can be interpreted as automatic activation of motor representations by the observed biological movement if a response is required at the same time as the movement is observed. If the go-signal is presented after the offset of movement stimuli, no such effect was present. As RTs in all congruent offset presentations are very similar to RTs in biological onset presentations, one could critically suggest, that it is the non-biological congruent condition, which inhibits responses in onset presentations. We would rather interpret these findings in terms of a ceiling effect of RTs in congruent offset presentations; here, subjects may not be capable to exhibit additional response facilitations over and above congruency from the biological type of movement. This further implies that the facilitative impact of movement observation on movement execution is transient and requires a close temporal relationship between the observed 
and executed movement. Taken together, the results of Experiment 1 show that the observation of irrelevant biological movement stimuli (i.e., tapping movements) can facilitate choice responses to auditory cues as opposed to kinematically matched non-biological dot stimuli. Importantly, response facilitation was only present if the auditory go-signal was coupled with movement observation.

In Experiment 1, we presented 8 to 11 movement cycles before the auditory cue. We assumed that the facilitation of response tendencies through the AOEM system would be more robust with an increasing number of observed tapping movements. However, Experiment 1 did not examine whether the observation of a short movement sequence would be sufficient to produce similar response facilitation as opposed to the observation of a long movement sequence. To clarify this issue, we conducted an additional experiment in which participants observed only one or two movement cycles of tapping movements. Otherwise, experimental procedures were identical to Experiment 1.

\section{Experiment 2}

3.1. Materials and methods

\subsubsection{Subjects}

Sixteen healthy subjects aged between 24 and 45 years (mean 31 years) participated in this experiment, half of them were male. As in Experiment 1 all were consistently right handed, had no history of neuropsychiatric disorder and had normal vision.

\subsubsection{Experimental procedure and task}

In the main measurement subjects observed sequences of visual stimuli comprising a resting hand and one or two short movements, respectively. Additionally they heard a tone, which they had to respond to as in the baseline measurement and in Experiment 1. We did not apply a fixed trial length as in Experiment 1. Instead, each trial started with the static hand presented for 2 to $3 \mathrm{~s}$ (200-ms steps). This was followed by one or two movements with $433 \mathrm{~ms}$ duration each. The tone was presented at onset or offset of the last movement. Trial duration varied between 2433 and 3916 ms. Otherwise, experimental procedures were identical to Experiment 1.

\subsubsection{Experimental design}

We applied a 2 (type of movement) $\times 2$ (congruency) $\times 2$ (presentation mode) $\times 2$ (number of stimuli) factorial design. The additional factor "number of stimuli" coded if the subject observed one or two movements, the other factors were adopted from Experiment 1. Each of the 16 conditions was applied 20 times; we therefore presented a total of 320 valid trials. We also applied 80 catch trials, i.e., $20 \%$ of all trials altogether. In these trials two movement sequences were shown but tones were omitted. This was done to avoid subjects' expectation that they have to respond after the second movement stimulus at the latest. All 400 trials were presented randomly in 10 blocks with 40 trials each, one block lasted approximately $2 \mathrm{~min}$.

\subsubsection{Statistical evaluation}

The analysis was performed as in Experiment 1. We proposed the same hypotheses as for Experiment 1. If any effect observed in Experiment 1 depended on prolonged repetitive stimulation it should not be present in Experiment 2.

\subsection{Results}

Error rate of all 16 subjects ranged from 0.8 to $10.5 \%$ (mean 3.5\%). Multiple responses and false single responses again comprised the highest amount of errors with $87 \%$ of all errors during onset presentations and $89 \%$ during offset presentations. In the baseline measurement responses took $415 \mathrm{~ms}$ on average (SD $74 \mathrm{~ms}$ ), which was statistically not different from $423 \mathrm{~ms}$ (SD $74 \mathrm{~ms}$ ) revealed in Experiment 1 (t-test, $\mathrm{p}=.76$ ). RTs from the main measurements of Experiment 1 and 2 averaged across conditions were 521 ms (SD 26 ms) and 488 ms (SD 43), 
respectively. Whereas RTs did not differ significantly (t-test; $p=.31$ ), standard deviations of Experiment 2 exceeded those of Experiment 1 (t-test; $p<.002$ ).

ANOVA results are given in Table 2. "Type of movement" did not reveal a significant effect during any presentation mode ("onset": biological 501 ms vs. dot 505 ms; "offset": 476 ms vs. 470 ms), whereas "congruency" was highly significant in both conditions ( $p$ <.001) ("onset": congruent $481 \mathrm{~ms}$ vs. incongruent 525 ms; "offset": 459 ms vs. $486 \mathrm{~ms}$ ) always pointing to shorter RTs with congruent stimuli. Additionally, the interaction between type of movement and congruency was significant in onset $(p=.029)$ but not in offset stimulation trials $(p=.670)$. During onset stimulation, RTs following biological stimuli were more strongly affected by congruency than those following non-biological. Fastest RTs were produced in congruent biological conditions (473 ms) whereas slowest RTs were produced in incongruent biological conditions $(528 \mathrm{~ms}$ ) with a difference of $55 \mathrm{~ms}$ (Scheffé-test, $\mathrm{p}<.001)$. The corresponding difference between congruent and incongruent RTs in non-biological conditions was 34 ms (Scheffétest, $\mathrm{p}<.001)$.

Table 2.

Results of analyses of variance, Experiment 2

Reaction times in milliseconds; means and standard deviations (SD)

\begin{tabular}{|c|c|c|}
\hline \multirow{3}{*}{ Conditions } & Onset presentation & \multirow[t]{2}{*}{ Offset presentation } \\
\hline & & \\
\hline & \multicolumn{2}{|c|}{ Biological Non-biological Biological Non-biological } \\
\hline Congruent & $473(79) \quad 488(95)$ & $461(91) \quad 457(91)$ \\
\hline Incongruent & $528(91) 522(89)$ & $490(91) \quad 482(79)$ \\
\hline \multicolumn{3}{|c|}{ Effects: $p$-values (F-values) } \\
\hline \multicolumn{2}{|c|}{ Type of movement $0.393(0.78)$} & $0.340(0.97)$ \\
\hline Congruency & $0.001(47.51)$ & $0.001(22.47)$ \\
\hline Interaction & $0.029(5.79)$ & $0.670(0.19)$ \\
\hline
\end{tabular}

Mean reaction times, standard deviations and p- and F-values for Experiment 2.

Mean RTs for all conditions and both experiments are illustrated in Fig. 2. 


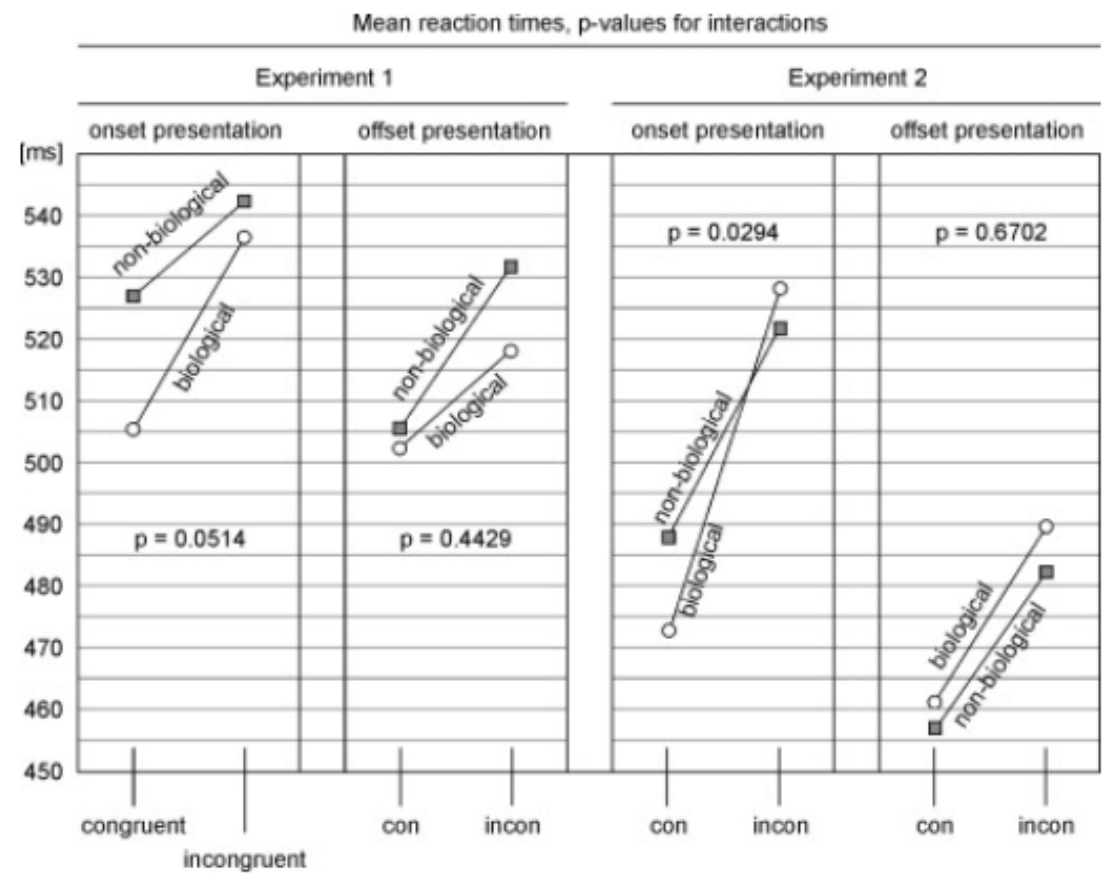

Fig. 2.

Mean RTs for the factors "type of movement" and "congruency" illustrated for Experiment 1 (left panel) and Experiment 2 (right panel) separately for onset presentations and offset presentations. $p$-Values of interactions as revealed by analyses of variance are plotted in the corresponding panels.

\subsubsection{Statistical comparison between experiments}

To compare compatibility effects between on- and offset presentations and between experiments, we calculated difference values of RTs between incongruent and congruent conditions separately for biological and non-biological trials in onset and offset presentations for both experiments. Thereby, the focus is laid on the size of the compatibility effect, which is supposed to be increased for biological as compared with non-biological trials, at the same time integrating both facilitative and inhibitory effects and eliminating divergent average RT-durations (of no interest in the present study) in both experiments. A 3-factors ANOVA with "Experiment", "presentation mode" and "type of movement" was calculated with RT-difference values as dependent variable.

There was an interaction between "type of movement" and "presentation mode" ( $p=.023)$ illustrated in Fig. 3. In the onset presentation mode the RT-benefit in trials with biological stimuli (43 ms) was larger than that in trials with non-biological stimuli ( $25 \mathrm{~ms}$ ) but this difference was just not significant (post-hoc Scheffé-test, $p=.064$ ). In contrast, in the offset presentation mode there was no difference between biological versus non-biological stimuli trials (posthoc Scheffé-test, $p=.959$ ).

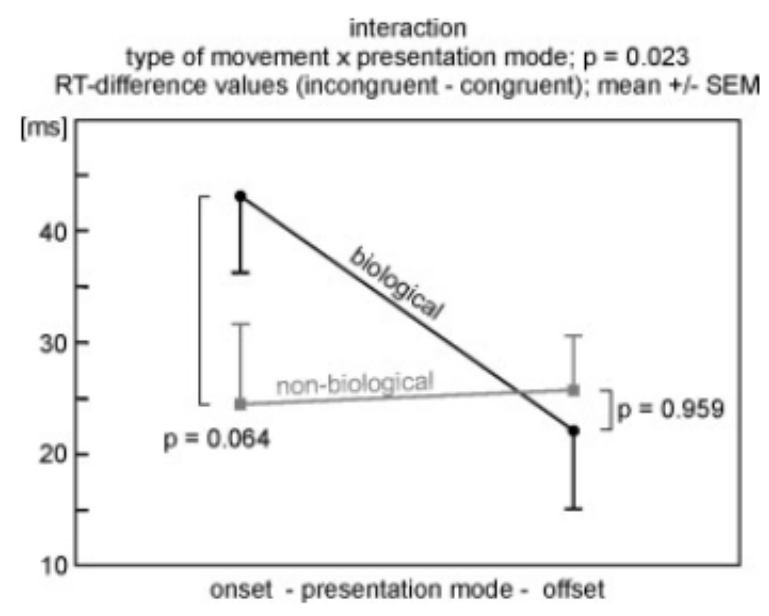

Fig. 3. 
Interaction between type of movement and presentation mode concerning RT-difference values (incongruent minus congruent) resulting from 3-way ANOVA (Experiment $\times$ presentation mode $\times$ type of movement). $p$-values related to pairwise comparisons result from post-hoc Scheffé-tests.

\section{General discussion}

RTs were faster to biological stimuli than to non-biological stimuli in congruent trials, but only if the auditory gosignal was presented at the onset of the observed movement. No facilitative effect of biological stimuli was observed with incongruent trials. In addition, the size of the compatibility effect was enlarged for biological trials in onset presentations as compared with onset non-biological trials and as compared with both movement conditions in offset presentations. These data suggest an automatic activation of the AOEM system with more efficient processing of spatially and temporally congruent biological movements as compared with non-biological stimuli on the one hand and an accompanying inhibition of responses in biological incongruent trials on the other hand. These results tie in with behavioural and neurophysiological studies showing that the observation of human biological agents is more effective in generating motor activation than compared with any other type of stimulus. For instance, the perception of a specific biological movement interferes more with the execution of an incongruent movement when it is performed by a person than when it is performed by a robotic agent (Castiello et al., 2002, Kilner et al., 2003 and Press et al., 2005). Transcranial magnetic stimulation (TMS) studies revealed that action observation results in an increase in corticospinal excitability as indexed by the amplitude of motor-evoked potentials (MEP) (Aziz-Zadeh et al., 2002, Fadiga et al., 1995 and Strafella and Paus, 2000), which reflects the response tendency of the subject to imitate the observed movement. Importantly, the increase in MEP amplitude was specific to those muscles, which are involved in executing the observed actions (Fadiga et al., 1995).

However, facilitation and inhibition of motor behaviour might occur only if the task requires an immediate response. Neuroimaging studies indicate stronger motor activation or resonance while observing human movements than while observing movements of a robotic or "virtual" agent, (Costantini et al., 2005, Perani et al., 2001 and Tai et al., 2004). It seems that observing biological movement optimally prepares the human motor system to execute the perceived movement. Apparently, such facilitation by biological stimuli is very transient and can only be observed "on-line", i.e., in simultaneous (onset) conditions. This is in concordance with our findings by means of whole-head magnetoencephalography (MEG), where evoked fields (Biermann-Ruben et al., 2008) as well as cortico-cortical synchronization (Kessler et al., 2006) revealed very transient differences of activations during processing of biological stimuli as compared with non-biological stimuli. Note that these stimuli were identical to the stimuli employed here (i.e., to a single repetition). Also, in an fMRI study using a very similar setup we failed to find stronger activation in the fronto-parietal MNS network, which could be partially due to the transient nature of the MNS activation (given that physical differences between biological and non-biological stimuli were controlled very thoroughly (Jonas et al., 2007b).

Finally, during longer lasting repetitive visual stimulations-requiring holding a movement in store until a go-signal appears-this effect is diminished (compare Experiment 1 ). This could be attributed to a progressive stable equilibrium between repeated stimulatory MNS-loops and reactive inhibitory processes aiming at suppressing inappropriate movement imitations. The latter is supported by the comparison of RTs in the different experiments: baseline RTs were very similar in Experiment $1(423 \mathrm{~ms})$ and $2(415 \mathrm{~ms})$, indicating that there were no differences attributable to the subject groups. RTs in the main measurements, however, ranged between 502 and $542 \mathrm{~ms}$ in Experiment 1 (mean: 521 ms; SD: 26 ms; range: $40 \mathrm{~ms}$ ) and 438 and 563 ms, respectively, in Experiment 2 (mean: $488 \mathrm{~ms}$; SD: $43 \mathrm{~ms}$; range: $125 \mathrm{~ms}$ ). Significantly smaller RT variability in Experiment 1 suggests that inhibitory and attentional processes due to repetitive visual stimulation dominate over processes probably mediated by AOEM.

\section{Final conclusion}

We conclude that RTs are specifically facilitated by irrelevant but congruent biological movement stimuli whereas they are inhibited by irrelevant but incongruent biological movement stimuli. This effect is very transient and can 
only be observed in simultaneous stimulation settings, i.e., when the cue, which codes the movement (here: tone) and the irrelevant visual movement stimulus are presented simultaneously. Contrary to our assumption, longstanding visual movement stimulation obscures the observed effects. We suggest that the facilitation and inhibition produced by biological stimuli in our setting is mediated by AOEM processes that automatically code observed motor actions into own motor actions, and which putative neural substrate is a human cortical MNS.

\section{References}

M. Annett

Left, right, hand and brain: The right shift theory

Lawrence Erlbaum Associates Ltd (1985)

L. Aziz-Zadeh, F. Maeda, E. Zaidel, J. Mazziotta, M. lacoboni

Lateralization in motor facilitation during action observation: A TMS study

Experimental Brain Research, 144 (1) (2002), pp. 127-131

K. Biermann-Ruben, K. Kessler, M. Jonas, H.R. Siebner, T. Bäumer, A. Münchau, A. Schnitzler

Right hemisphere contributions to imitation tasks

European Journal of Neuroscience, 27 (2008), pp. 1843-1855

B.I. Bertenthal, M.R. Longo, A. Kosobud

Imitative response tendencies following observation of intransitive actions

Journal of Experimental Psychology: Human Perception and Performance, 32 (2) (2006), pp. 210-225

M. Brass, H. Bekkering, A. Wohlschläger, W. Prinz

Compatibility between observed and executed finger movements: Comparing symbolic, spatial, and imitative cues

Brain and Cognition, 44 (2) (2000), pp. 124-143

U. Castiello, D. Lusher, M. Mari, M. Edwards, G.W. Humphreys

Observing a human or a robotic hand grasping an object: Differential motor priming effects

W. Prinz (Ed.), Common mechanisms in perception and action, Oxford University Press (2002)

M. Costantini, G. Galati, A. Ferretti, M. Caulo, A. Tartaro, G.L. Romani, S.M. Aglioti

Neural systems underlying observation of humanly impossible movements: An FMRI study

Cerebral Cortex, 15 (11) (2005), pp. 1761-1767

G. di Pellegrino, L. Fadiga, L. Fogassi, V. Gallese, G. Rizzolatti

Understanding motor events: A neurophysiological study

Experimental Brain Research, 91 (1) (1992), pp. 176-180

L. Fadiga, L. Fogassi, G. Pavesi, G. Rizzolatti

Motor facilitation during action observation: A magnetic stimulation study

Journal of Neurophysiology, 73 (6) (1995), pp. 2608-2611

P.M. Fitts, C.M. Seeger

S-R compatibility: Spatial characteristics of stimulus and response codes

Journal of Experimental Psychology, 46 (3) (1953), pp. 199-210

L. Fogassi, P.F. Ferrari, B. Gesierich, S. Rozzi, F. Chersi, G. Rizzolatti

Parietal Lobe: From Action Organization to Intention Understanding

Science, 308 (5722) (2005), pp. 662-667

V. Gallese, L. Fadiga, L. Fogassi, G. Rizzolatti

Action recognition in the premotor cortex

Brain, 119 (Pt 2) (1996), pp. 593-609 
V. Gallese, C. Keysers, G. Rizzolatti

A unifying view of the basis of social cognition

Trends in Cognitive Sciences, 8 (9) (2004), pp. 396-403

\section{A.G. Greenwald}

On doing two things at once: Time sharing as a function of ideomotor compatibility

Journal of Experimental Psychology, 94 (1) (1972), pp. 52-57

\section{R. Hari, N. Nishitani}

From viewing of movements to imitation and understanding of other persons' acts: MEG studies of the human mirror-neuron system N. Kanwisher, J. Duncan (Eds.), Functional Neuroimaging of Visual Cognition. Attention and Performance, University Press, Oxford (2004)

\section{Iacoboni, M. Dapretto}

The mirror neuron system and the consequences of its dysfunction

Nature Reviews Neuroscience, 7 (12) (2006), pp. 942-951

\section{W. James}

The principles of psychology

Dover, New York (1890)

M. Jonas, K. Biermann-Ruben, K. Kessler, R. Lange, T. Bäumer, H.R. Siebner, A. Schnitzler, A. Münchau Observation of a finger or an object movement primes imitative responses differentially

Experimental Brain Research, 177 (2) (2007), pp. 255-265

M. Jonas, H.R. Siebner, K. Biermann-Ruben, K. Kessler, T. Bäumer, C. Büchel, A. Schnitzler, A. Münchau Do simple intransitive finger movements consistently activate frontoparietal mirror neuron areas in humans? Neuroimage, 36 (Suppl. 2) (2007), pp. T44-T53

K. Kessler, K. Biermann-Ruben, M. Jonas, H.R. Siebner, T. Bäumer, A. Münchau et al. Investigating the human mirror neuron system by means of cortical synchronization during the imitation of biological movements Neuroimage, 33 (2006), pp. 227-238

J.M. Kilner, Y. Paulignan, S.J. Blakemore

An interference effect of observed biological movement on action

Current Biology, 13 (6) (2003), pp. 522-525

S. Kornblum, T. Hasbroucq, A. Osman

Dimensional overlap: Cognitive basis for stimulus-response compatibility--a model and taxonomy

Psychological Reviews, 97 (2) (1990), pp. 253-270

L. Koski, M. Iacoboni, M.C. Dubeau, R.P. Woods, J.C. Mazziotta

Modulation of cortical activity during different imitative behaviors

Journal of Neurophysiology, 89 (1) (2003), pp. 460-471

D. Perani, F. Fazio, N.A. Borghese, M. Tettamanti, S. Ferrari, J. Decety, M.C. Gilardi Different brain correlates for watching real and virtual hand actions

Neuroimage, 14 (3) (2001), pp. 749-758

\section{Press, G. Bird, R. Flach, C. Heyes}

Robotic movement elicits automatic imitation

Brain Research Cognitive Brain Research, 25 (3) (2005), pp. 632-640

\section{G. Rizzolatti}

Understanding the actions of others

N. Kanwisher, J. Duncan (Eds.), Functional neuroimaging of visual cognition. Attention and performance, University Press, Oxford (2004)

G. Rizzolatti, L. Fadiga, V. Gallese, L. Fogassi

Premotor cortex and the recognition of motor actions

Brain Research Cognitive Brain Research, 3 (2) (1996), pp. 131-141 
J.R. Simon

The effects of an irrelevant directional cue on human information processing

R.W. Proctor, T.G. Reeve (Eds.), Stimulus-response compatibility, Elsevier (1990)

A.P. Strafella, T. Paus

Modulation of cortical excitability during action observation: A transcranial magnetic stimulation study

Neuroreport, 11 (10) (2000), pp. 2289-2292

Stürmer, B. (1997). Organisationsprinzipien an der Schnittstelle zwischen Wahrnehmung und Handlung: Kompatibilitätseffekte unter Verwendung dynamischer Reiz- und Reaktionseigenschaften. München.

B. Stürmer, G. Aschersleben, W. Prinz

Correspondence effects with manual gestures and postures: A study of imitation

Journal of Experimental Psychology: Human Perception and Performance, 26 (6) (2000), pp. 1746-1759

Y.F. Tai, C. Scherfler, D.J. Brooks, N. Sawamoto, U. Castiello

The human premotor cortex is 'mirror' only for biological actions

Current Biology, 14 (2) (2004), pp. 117-120 